\title{
Two-dimensional colloidal systems in time-dependent magnetic fields
}

\author{
How to define crystallinity in $2 \mathrm{D}$ on a local scale \\ Patrick Dillmann, Georg Maret, and Peter Keim ${ }^{\mathrm{a}}$ \\ Universität Konstanz, Fachbereich Physik, 78467 Konstanz, Germany
}

\begin{abstract}
We use super-paramagnetic colloidal particles confined by gravitation to a flat water-air interface as a model system to study the non-equilibrium liquid-solid phase transition in two dimensions. The system temperature is adjustable by changing the strength of an external magnetic field perpendicular to the water-air interface. Increasing the magnetic field on a timescale of milliseconds quenches the liquid to a strongly super-cooled state. If the system is cooled down out of equilibrium the solidification differs drastically from the equilibrium melting and freezing scenario as no hexatic phase is observable. The system solidifies to a polycrystalline structure with many grains of different orientations. Since the local closed packed order in two dimensions is sixfold, in both the fluid and the crystalline state, sensitive measures have to be developed. In the present manuscript we compare different methods to identify crystalline cluster locally and motivate the threshold values. Those are chosen in comparison with the isotropic fluid on one hand and large mono-crystals in thermal equilibrium on the other hand. With the given criteria for crystalline cluster the cluster are found not to be circular and fractal dimensions of the grains are given.
\end{abstract}

\section{Introduction}

Since the work of Peierls [1] and Mermin and Wagner [2,3] it is known that strictly speaking no crystals exist in systems with dimension $d<3$ at finite temperature. In general the significance of thermal fluctuations is increased when the dimension of a system is decreased. Crystal lattices with dimension $d<3$ are thermally unstable due to long wavelength phonon modes. As a consequence long range translational order does not exist in $1 \mathrm{~d}$ whereas in $2 \mathrm{~d}$ fluctuations around lattice sites diverge logarithmicaly in the crystalline phase [4]. Likewise this is also detectable with dynamic functions [5]. The slow logarithmic divergence in two dimensions leads to crystals which possess only a quasi long-range translational order but perfect long-range orientational order. Given the existence of $2 \mathrm{~d}$ crystals it has been a matter of debate over

\footnotetext{
a e-mail: peter.keim@uni.kn
} 
decades how a two-dimensional crystal melts. While the liquid to crystal transition in three-dimensional systems is usually a first order transition, the situation in twodimensional systems is found to be more complex. Grain-boundary induced melting [6-8] or condensation of geometrical defects $[9,10]$ suggest a first order phase transition, while the theory of John M. Kosterlitz, David J. Thouless, Bertrand Halperin, David R. Nelson, and Allan P. Young [11-17], the so called KTHNY theory, predicted a melting process via two continuous phase transitions with an intermediate phase. The intermediate phase appears due to the fact that the translational and orientational symmetries are broken at different temperatures. The first phase transition at temperature $T_{m}$ is associated with destroying the discrete translational symmetry. The intermediate phase is named hexatic, based on the remaining sixfold quasi long range orientational order. If the orientational order degenerates to short range at temperature $T_{i}>T_{m}$, a second phase transition from the hexatic to the isotropic liquid occurs. According to the KTHNY theory, the different symmetries are affiliated with the occurrence of different topological defects, namely dislocations and disclinations.

In simulations [18-26] the results were controversial for a long time but meanwhile the hexatic phase is well established. In experiments the hexatic phase is observed, too [27-36] but in both, experiments ans simulations the nature of the transitions is debated: indications of first order transitions are reported in a colloidal system with screened coulomb interactions [30,31] and in di-block copolymer systems $[34,35]$. Like in simulations, a phase coexistence is usually interpreted as first order signature. KTHNY theory is a melting theory starting from large single crystals but of course a thermodynamic phase should be independent of the history of the matter and cooling and heating cycles should yield the same results. Wang et al. reported polycrystalline domains during cooling in a system of diameter-tunable microgel spheres at finite cooling rates [37]. Indeed, cooling rates have to be small such that critical fluctuations of a continuous order phase transition can switch the symmetry globally. In a system of particles with dipolar interaction, where the monolayer is confined to an air-water interface as described below, we measured the softening of elasticity to be completely consistent with renormalization group calculations [38]. We found both transitions, the crystal to hexatic and the hexatic to isotropic fluid transition to be continuous during melting and freezing if the system is always kept in thermal equilibrium. Indeed, if the cooling rate is very slow we do observe homogeneous large single domain crystals in the field of view $[39,40]$, implying that KTHNY theory also holds for freezing. But, as we summarise in this minireview, cooling the system rapidly from the isotropic liquid to the crystalline state, we find a polycrystalline sample without a signature of a hexatic phase during crystallization [41]. Obviously the mechanism of annihilation of topological defects is too slow for crystallization if the system is supercooled on short timescales.

In 3 dimensions, supercooled fluids nucleate homogeneously or heterogeneously from the isotropic phase to the periodic structure. The underlying phase transition is a first order transition where phase equilibrium exists and the nucleation process has been intensely studied [42-56]. The most widely used concept, the Classical Nucleation Theory (CNT), attempts to describe this complex process by treating the nuclei as compact spheres that grow if their size exceeds a critical value [42-44]. However, experiments on hard sphere colloidal systems $[48,49,52]$ differ from the predictions of critical nucleation theory and the CNT calculation underestimates the nucleation rate by many orders of magnitude [57]. In computer simulation in 3D [58] and for phase-field-crystal calculations in 2D [59], a precursor-mediated two-step crystallization was observed. In experiments in $2 \mathrm{D}$ with attractive particle interactions $[60,61]$ such precursors are found, too, but might be explained with sublimation rather than nucleation. 


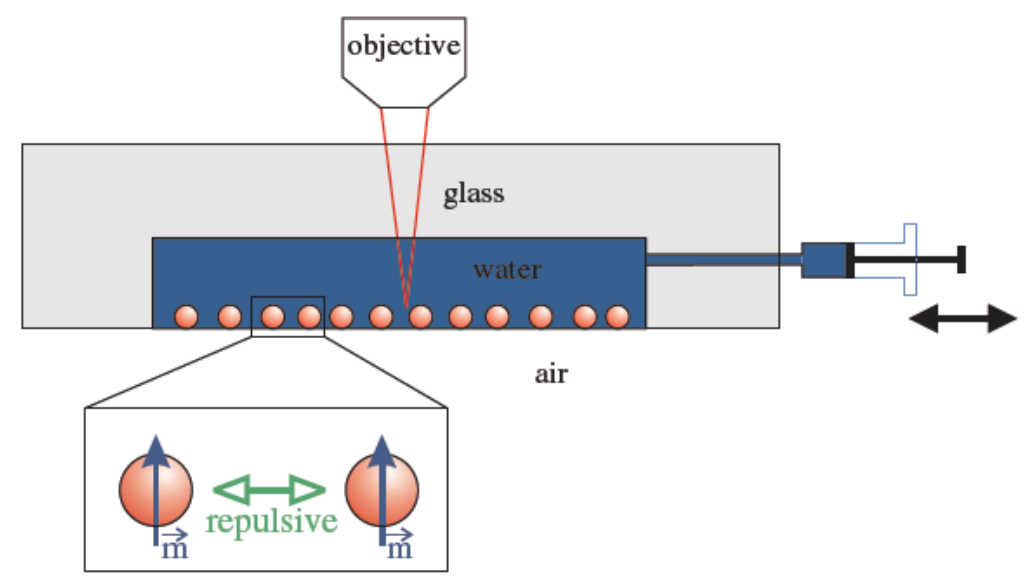

Fig. 1. Sketch of the sample cell to make 2D colloidal crystals. Magnetic colloids are confined to the water/air interface and a vertical magnetic field induces a repulsive dipole interaction. A time dependent magnetic field can quench the monolayer from a disordered isotropic phase rapidly to a polycrystalline state.

In 3d, a bond order parameter based on spherical harmonic functions is used to identify local grains $[49,62,63]$. The locally favored structures for systems with isotropic particles interactions are icosahedral in the fluid [64,65]. This structure is not compatible with long range periodic order and it is usually not a problem to distinguish fluid and crystalline phases but the competing crystal structures like fcc, hcp, rcp, or bcc. In $2 \mathrm{~d}$ our experiment with dipolar particle interaction showed that the underlying equilibrium phase diagram does not have phase coexistence and the question arises if and how this affects the nucleation process of the supercooled fluid. The locally favored structure is sixfold in both, the fluid and the crystalline phase such that long range order is possible to built up with locally favored structures of the fluid. Hence, sensitive measures have to be developed to study nucleation and distinguish crystalline and fluid structures locally. The aim of this minireview is to describe, how such measures are defined and how the threshold values are determined. After a brief introduction to the experimental setup, we describe the time dependent increase of the global order after a temperature quench and verify that the final structure is a polycrystalline one. The main part of this minireview is the description of the criteria for crystal-like grains. The criteria which work best are based on the the local orientational order, the bond length variations, and the relative orientation of neighboring particles. The threshold values which we introduce are discussed in comparison with homogeneous equilibrium phases. With this criteria the shape and the fractal dimension of the crystalline grains are analysed.

\section{Experimental setup}

Our experimental setup consists of $4.5 \mu \mathrm{m}$ diameter spherical colloids confined by gravity to the flat water-air interface of a hanging droplet, see Fig. 1 or [66]. The $8 \mathrm{~mm}$ diameter sample cell contains about $\approx 300000$ particles which are doped with iron oxide nanoparticles making them superparamagnetic. A magnetic field is applied perpendicular to the $2 \mathrm{D}$ water-air interface which induces a magnetic moment $\mathbf{m}=\chi \mathbf{H}$ in each particle. This leads to a repulsive dipole-dipole interaction proportional to $\mathrm{m}^{2} / \mathrm{r}^{3}$ between beads at distance $r$. The interaction strength $\Gamma$ is defined as the ratio between repulsive magnetic interaction and thermal energy $\Gamma \propto E_{\text {mag }} / k_{B} T \propto \chi^{2} H^{2} / r^{3} \cdot 1 / k_{B} T$ 
and acts like an inverse temperature. The experiments are carried out at constant ambient temperature but the effective temperature is varied by the magnetic field $\mathbf{H}$ which is physically equivalent. At high magnetic fields we observe a hexagonal crystal if the system is kept always in thermal equilibrium while at low fields Brownian motion dominates, the system is disordered and particles diffuse freely throughout the $2 \mathrm{D}$ plane. The melting temperature in units of the interaction parameter between crystal and hexatic is about $\Gamma_{m}=60$ and the transition temperature between hexatic and isotropic liquid is about $\Gamma_{i}=57.5$ [67]. We can monitor the different states using a CCD-camera looking at a $1 \mathrm{~mm}^{2}$ spot with a frame rate of $10 \mathrm{fps}$ containing several thousand particles. Images are analysed and particles are tracked using digital image analysis. This way we record the trajectory of all particles and obtain the whole phase-space information. Thanks to a computer controlled regulation of the sample volume we keep the water-air interface flat such that number density inhomogeneities are less then $0.1 \%$ over several months. Using magnetic colloids has the advantage that the pair-potential is known precisely and can be varied in strength by the applied magnetic field. This way, the (inverse) system temperature can be varied on a time scale $10^{4}$ times faster compared to intrinsic time scales like the Brownian time which is of the order of $50 \mathrm{sec}$. The power supply switches the magnetic field within $5 \mathrm{~ms}$. During that time, diffusion would lead to an average translation of $50 \mathrm{~nm}$ which is comparable to the resolution in particle position by video microscopy. For all the data shown, the quench is repeated at least ten times and the results are averaged for those independent runs. After a quench, the system is remelted and equilibrated for a few days bevor the next measurement.

\section{Time dependent crystallization}

To identify the degree of orientational order we calculate the sixfold bond orientation parameter which assigns to every particle $k$ at position $\mathbf{r}$ a complex number. The phase and the magnitude of this complex number is marking the local orientational order given by the $N_{j}$ nearest neighbours with bond orientation $\theta_{j k}$ to a fixed reference axis.

$$
\psi\left(\mathbf{r}_{k}\right)=\psi_{k}=\frac{1}{N_{j}} \sum_{j} e^{i 6 \theta_{j k}} .
$$

The phase of $\psi_{k}$ marks the absolute orientation of the neighbours in the sixfold orientational space and the magnitude $0 \leq m_{6_{k}}=\left|\psi_{k}\right| \leq 1$ measures the deviation of perfect hexagonal order. The global orientational order of an ensemble of $N$ particles in the field of view is given by

$$
\Psi^{\text {global }}=\left|\frac{1}{N} \sum_{i=1}^{N} \psi_{k}\right|
$$

whereas an average local order of the ensemble is given by

$$
\Psi^{\text {local }}=\frac{1}{N} \sum_{i=1}^{N}\left|\psi_{k}\right| .
$$

Figure 2 shows the averaged local and global orientational order parameter as function of time after a quench for different quench-depths. The deeper the quench, the faster is the increase in orientational order, as expected. Within the duration of measurements of $10 \mathrm{~min}$, the orientational order does not saturate but for the deepest quench to 

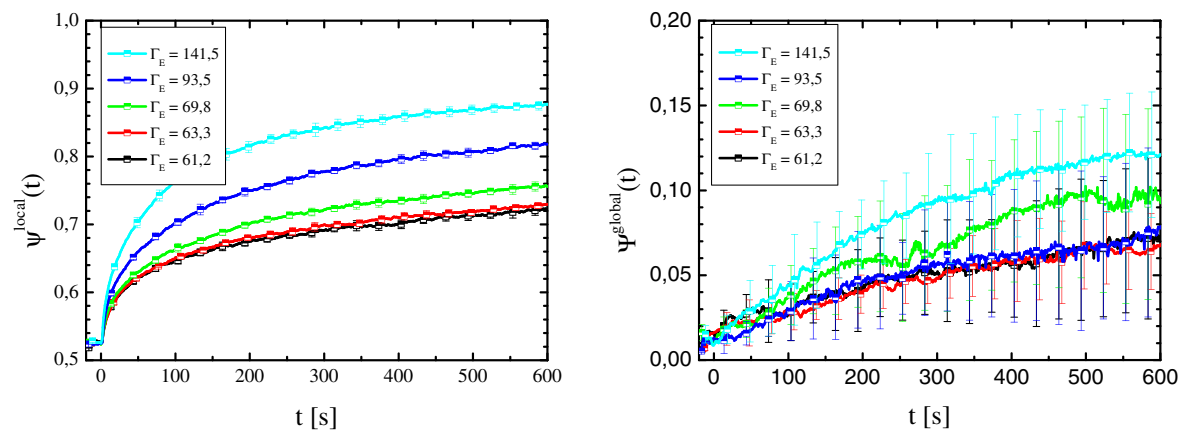

Fig. 2. Local (left) and global (right) orientational order parameter as function of time after a quench for different quench-depth. Note the different y-axes. The orientational order increases in all cases as function of time but the global orientation is much smaller compared to the local one. This indicates that the system crystallize into several grains of different orientation. The error bars are the variance of at least ten independent quenches to the same value.

$\Gamma_{E}=141$, the local orientational order approaches $90 \%$. This is in contrast to the global orientational order where the deepest quench increases to a degree of about $10 \%$ within $10 \mathrm{~min}$. This clearly indicates that the system does not crystallize into a monocrystal but into several grains of individual orientation. This is furthermore seen in a snapshot in real space where the magnitude of the local director field $m_{6_{k}}=\left|\psi_{k}\right|$ is plotted, which is zero for perfect five- or sevenfold neighbored particles and one for perfect sixfold ones. In Fig. 3, the color code ranges from blue $\left(m_{6}=0\right.$, low orientational order) via yellow to red $\left(m_{6}=1\right.$, high orientational order). The system is obviously polycrystalline and grains of different orientation of the lattice are visible. As can be seen in the bottom of Fig. 3 solely the magnitude of the bond order field is not sufficient to identify crystalline cluster since the border between order and disordered areas are not sharp but rather washed-out. In the following section we discuss which criteria work best to identify local grains.

\section{Local criteria for crystallinity and their comparison with homogeneous phases in equilibrium}

As mentioned in the introduction, the phase transitions crystalline to hexatic and hexatic to isotropic are continuous transitions. Those continuous phase transitions are characterized by the absence of a phase equilibrium and the absence of latent heat. The system switches the symmetry of the appropriate order parameter on all length scales if the transition temperature is crossed. To exclude phase coexistence, Strandburg et al. introduced the bond angular stiffness being the magnitude squared of the global bond order parameter $\chi_{a n g}=\left\langle\left|\Psi_{\text {global }}\right|^{2}\right\rangle[68]$. In the original manuscript, this quantity was named nearest-neighbor bond-angular susceptibility, a terminus which is nowadays usually used for the variance of the bond order parameter defined as $\chi_{6}=\left\langle\left|\Psi^{2}\right|\right\rangle-\langle|\Psi|\rangle^{2}[36,37,67]$.

A finite size analysis of the probability distribution $P\left(\chi_{a n g}\right)$ may distinguish between a homogeneous phase and phase equilibrium. A monomodal distribution of the bond angular stiffness indicates a homogeneous phase, whereas a bimodal distribution indicates a phase coexistence of ordered and disordered regions in different subsystems of the same size. In Fig. 4 the finite size analysis of the bond angular stiffness is plotted for a system which has been always in thermal equilibrium. The upper row 

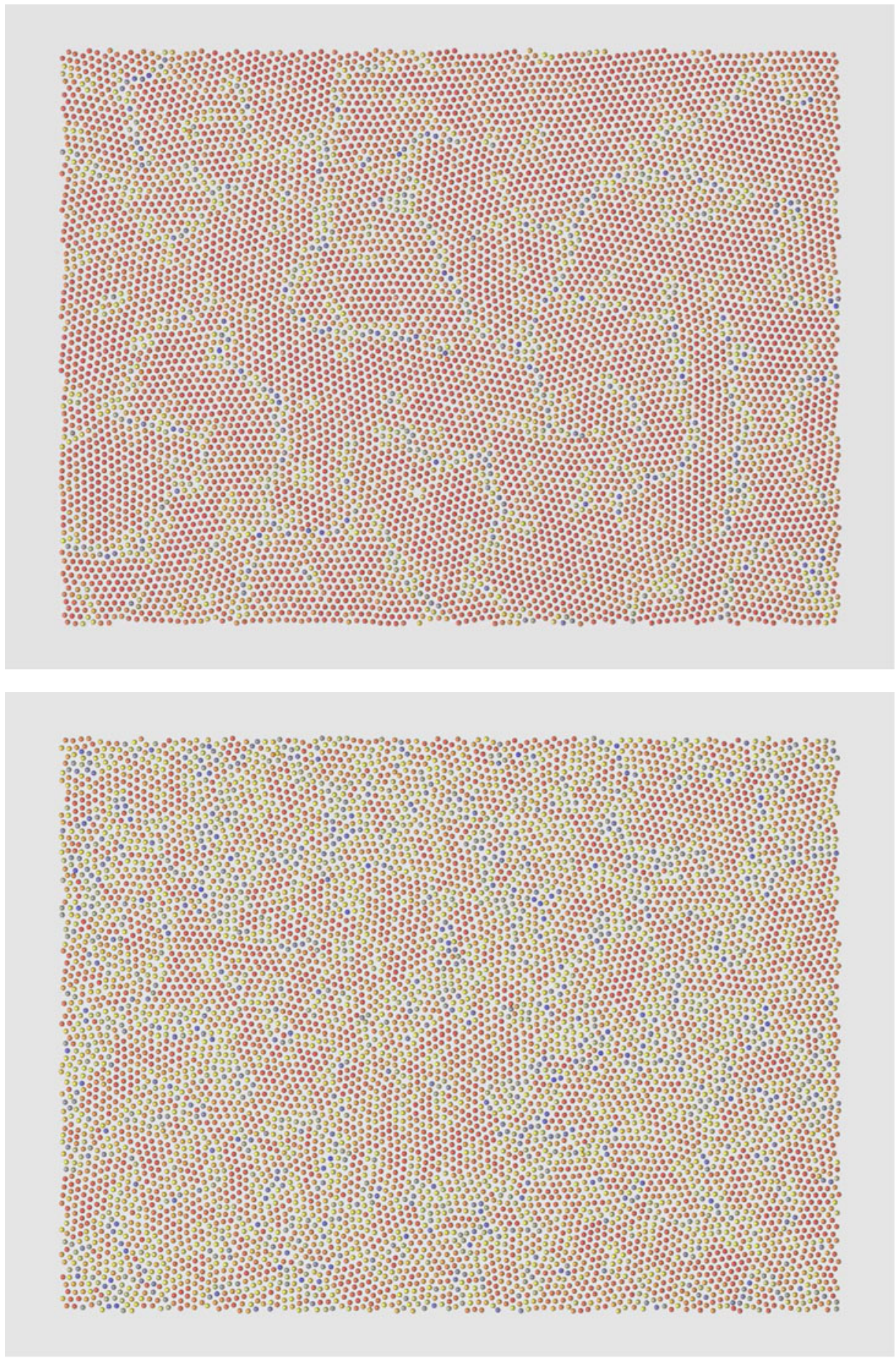

Fig. 3. Magnitude of the local bond order color coded in real space ten minutes after the quench. Blue corresponds to low orientational order $\left(m_{6}=0\right)$ and red to high orientational order $\left(m_{6}=1\right)$. Whereas for the deep quench to $\Gamma_{E}=141$ most of the particles arrange in crystalline grains separated by grain boundaries (top) the system did not yet crystallize completely for the low quench to $\Gamma_{E}=68$ (bottom). 

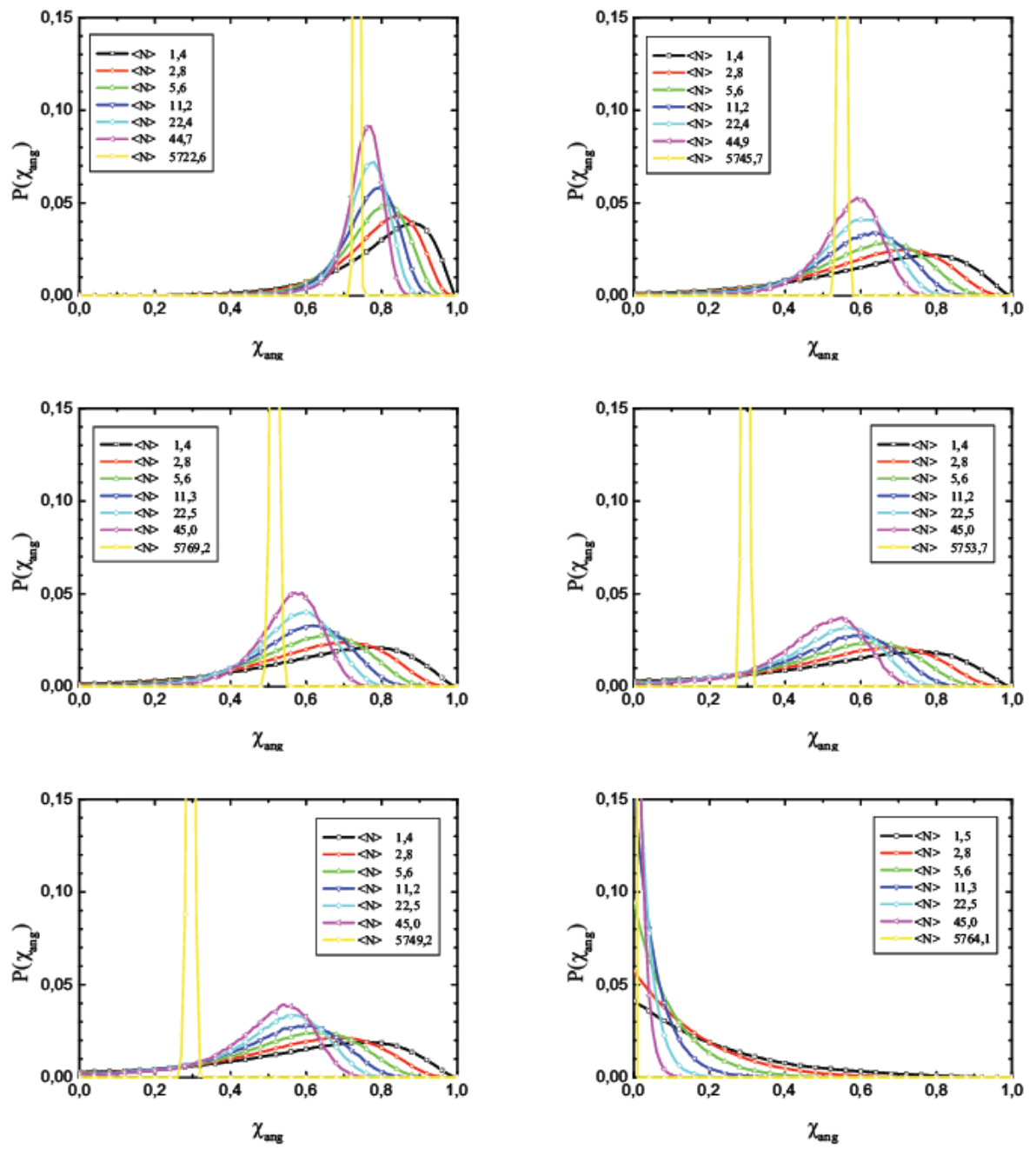

Fig. 4. Angular susceptibility of the crystalline phase (upper row), the hexatic phase (middle row) and the isotropic fluid phase (lower row) in equilibrium. Subsystems of decreasing size are analyzed and in all phases a monomodal distribution is found indicating a homogeneous phase without phase equilibrium.

shows monocrystalline ensembles deep in the crystalline phase at $\Gamma=133$ (left) and close to the hexatic phase at $\Gamma=62.2$ (right) the middle row shows ensembles in the hexatic phase at $\Gamma=59.6$ (left) and $\Gamma=57.8$ (right), and the lower row shows ensembles in the fluid phase close to the hexatic - isotropic transition at $\Gamma=56.4$ (left) and very deep in the fluid at $\Gamma=5.9$ (right). For smaller subwindows, where $\langle N\rangle$ is the number of particles in the subwindow, the distribution of the bond angular stiffness broadens due to decreasing statistics within the small subsystems and the maximum shifts to larger values since long range distortions are cut off. But the probability distribution is always monomodal. Decreasing the interaction parameter, so increasing system temperature, the maxima in the distribution shifts to low ordered values, as expected. For comparison the largest system size with about $\langle N\rangle=5700$ analysed particles in the field of view is plotted, too. The melting scenario in an 
equilibrium system shows homogeneous orientational bond stiffness consistent with KTHNY-melting and without any indications of phase equilibrium.

To analyze the time dependent behaviour of crystallization after a temperature quench below the melting temperature $\left(=\right.$ above $\left.\Gamma_{M}\right)$, Fig. 5 shows the bond angular stiffness for three different times. The upper graph is still in the fluid phase at $\Gamma=13$ and shows qualitatively the same behaviour as Fig. 4. The middle graph shows the distribution of $\chi_{a n g} 50 \mathrm{sec}$ after a quench to $\Gamma_{E}=141$ and the lower graph is $580 \mathrm{sec}$ after the quench. The curves are averaged for 100 consecutive timesteps in an interval of about $10 \mathrm{sec}$. Especially $50 \mathrm{sec}$ after the quench, a bimodal distribution is found for small subwindows with $\langle N\rangle=1.4$ and 2.8. The maxima of the probability distributions are at $\chi_{a n g}=0$ and $\chi_{a n g} \approx 0.8$ indicating heterogeneous local orientational order. $580 \mathrm{sec}$ after the quench, small subwindows show quite large local order with maxima between of $\chi_{\text {ang }} \approx 0.7$ and $\chi_{a n g} \approx 0.85$ but the largest window with $\langle N\rangle \approx 5700$ particles is peaked at vanishing or very small bond order stiffness $\chi_{\text {ang }}<0.05$. Consistent with Fig. 2 and Fig. 3 this indicates polycristalline order. At intermediate times, the bimodal distribution confirms, that areas with crystalline local order and areas with fluidlike order are present at the same time. Of course, this should not be named a phase equilibrium since the system is quenched rapidly out of thermal equilibrium.

Another way to analyze the local orientational order was suggested by Larsen and Grier [71]. It takes the magnitude of the local bond order field $m_{6_{k}}=\left|\psi_{6}\left(\mathbf{r}_{\mathbf{k}}\right)\right|$ and projects it to the mean of the local bond order field given by the nearest neighbours.

$$
n_{6_{k}}=\left|\psi_{6_{k}}^{*} * 1 / N_{l} \sum_{l} \psi_{6_{l}}\right|
$$

It takes the second nearest neighbors into account and determines how the orientation of the center particle fits into the orientational field of its neighbor particles. Since it is a projection $n_{6} \leq m_{6}$ and $n_{6}+m_{6} \leq 2$. In [71] an uni-modal distribution was found even if real space images showed a dilute liquid (or gas) phase and dense crystalline flakes implying the existence of an attractive interaction between particles, whereas in [31] a bimodal distribution is reported next to the isotropic-hexatic as well to the hexatic-crystalline transition. Particles in the $m_{6}-n_{6}$-plane with $m_{6}+n_{6}>1$ were identified to be crystal-like particles. In [67] we showed i) the absence of a bimodal distribution and ii) the weak dependence of the local bond-order field above and below the melting temperatures. This indicates continuous phase transitions for our system if kept always in equilibrium, consistent with KTHNY theory.

Figure 6 shows the probability distribution $220 \mathrm{sec}$ after a quench from $\Gamma=13$ to $\Gamma=63$ in the $m_{6}-n_{6}$-plane. The distribution is clearly bimodal with highly sixfold particles (upper right corner) and poorly sixfold particles (lover left corner). But to identify crystalline cluster it is not sufficient to investigate the magnitude of the local bond order field.

The bond length has to bee taken into account too preclude particles which have sixfold orientational symmetry but the neighbours are not sitting at the corners of a hexagon (see Fig. 7 right). This way not only the direction but the periodicity of the crystal is used to identify crystalline grains. Furthermore, the complex phase of the local bond order parameter has to be compared between neighboring particles to distinguish between crystalline cluster of different orientation.

Neighboring particles do belong to the same crystalline cluster if they fulfill those three criteria:

- The magnitude of the local bond order field $m_{6_{k}}$ must exceed a threshold value $m_{T}$ for both neighboring particles. 

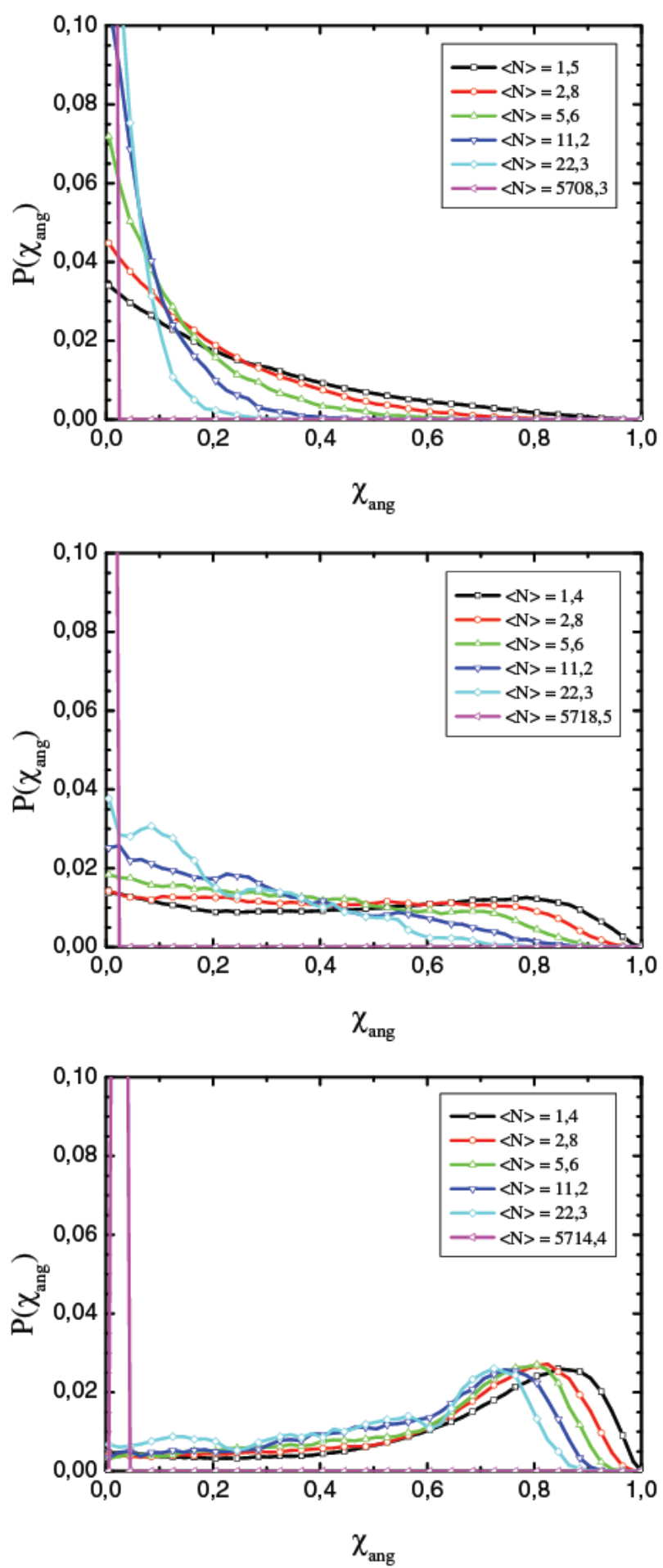

Fig. 5. Subsystem analysis of the angular susceptibility for a quenched system from $\Gamma=13$ to $\Gamma_{E}=141$ for different times. The upper plot shows the distribution before the quench, the middle plot $50 \mathrm{sec}$ and the bottom plot $580 \mathrm{sec}$ after the quench. 


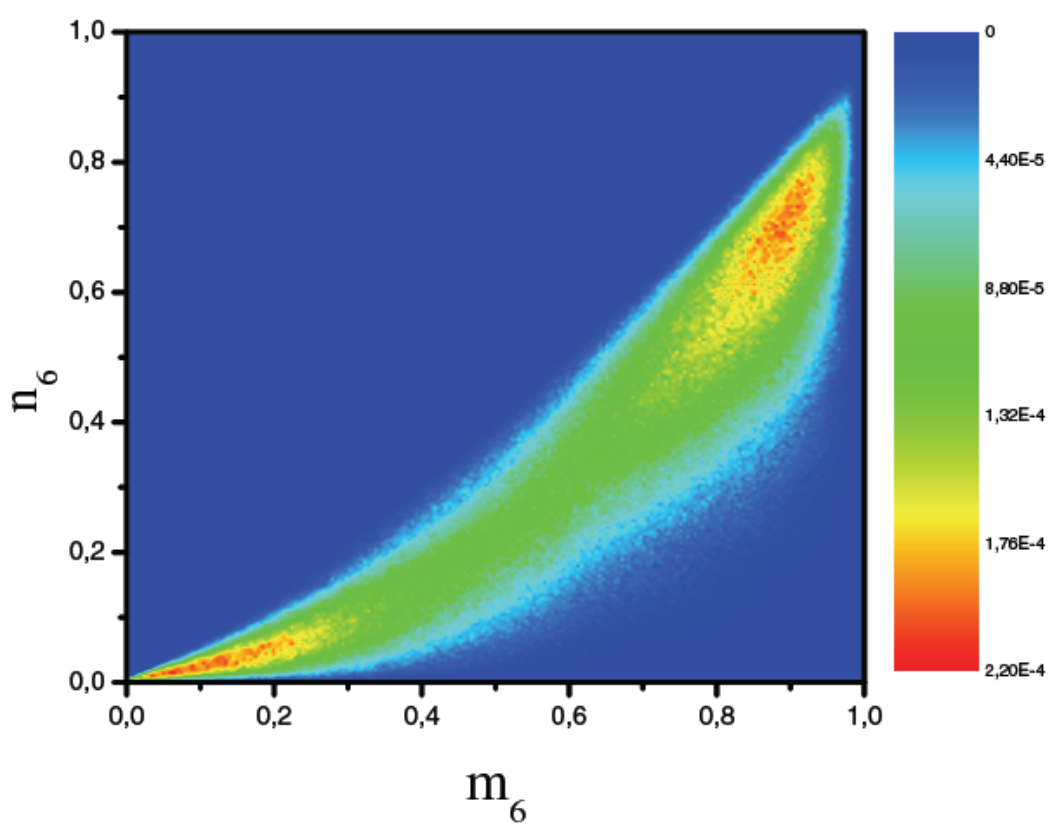

Fig. 6. The color code measures the frequency of locally ordered particles in the m-n-plane $220 \mathrm{~s}$ after a (inverse) temperature quench from $\Gamma=13$ (deep in the fluid phase) to $\Gamma=63$ in the crystalline state. A bimodal distribution of crystalline-like particles with high local order (upper right corner) and fluid-like particles with low local order (lower left corner) is found.
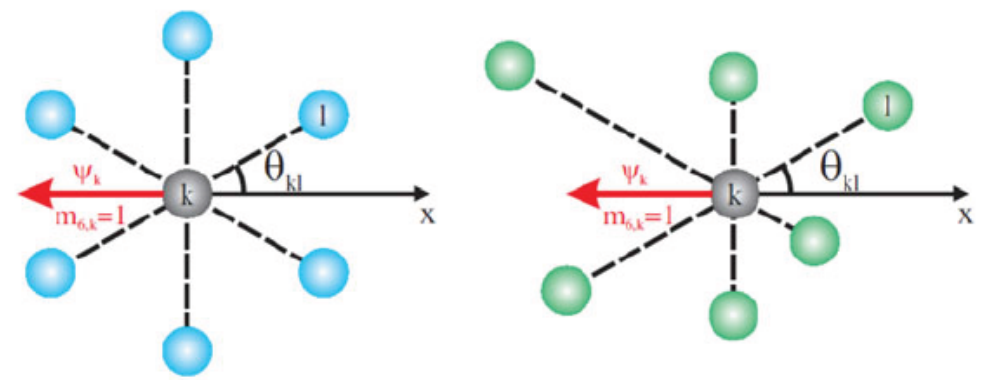

Fig. 7. Two different configurations, where the bond order parameter has the same magnitude.

- The bond length deviation $\Delta\left|l_{k l}\right|$ of neighboring particles $k$ and $l$ is less than a threshold value $l_{T}$.

- The variation in bond orientation $\Delta \Theta=\left|\psi_{k}-\psi_{l}\right|$ of neighboring particles $k$ and $l$ must be less than a threshold value $\Theta_{T}$ in sixfold space (less than $\Theta_{T} / 6$ in real space).

The threshold values have to be optimized according to the three criteria. First, a large defect free monocrystal of an equilibrated system has to be identified as a single grain. Here it is crucial that $\Theta_{T}$ is not too small and $m_{T}$ is not too large otherwise long wavelength fluctuations lead to a splitting of the crystal into subdomains. Second, even deep in the isotropic fluid phase for $\Gamma>10$ more than $50 \%$ of the particles have six nearest neighbours as determined by Voronoi tessellation (see Fig. 9). Therefore $l_{T}$ should be small and $m_{T}$ preferably large. A third criterion is that the grains identified 
with the computer algorithm reproduces the grains identified by eyes. The human brain is quite developed in pattern recognition and can easily distinguish between an artefact or tolerable perturbations since it compares the patterns a) parallel and globally and b) with former data. The optimization of the threshold parameters is done in comparison with the equilibrated system.

Figure 8 shows the probability distribution of $m_{6}$ (upper graph), the distribution of the bond length variation $\Delta l$ (middle graph) and the bond orientation difference $\Delta \Theta$ of neighboring particles (lower graph) in histograms of equilibrated systems. The interaction strength ranges from very fluid values to those deep in the crystalline phase.

1. $P\left(m_{6}\right)$ has a pronounced maximum at $m_{6}>0.9$ in the crystalline phase. With decreasing interaction strength the maximum decreases and shifts to lower values of $m_{6}$. In the hexatic phase and even in the fluid phase at $\Gamma=50$, the maximum is close to $m_{6} \approx 0.9$. For $\Gamma=39.5$ in the fluid phase a shoulder appears at $m_{6} \approx 0.35$. The shoulder grows to a maximum for very fluid phases and the maximum at $m_{6}>0.8$ has disappeared.

2. For decreasing interaction strength particles fluctuate increasingly around their lattice sites in the crystal. Since the particle pair potential is purely repulsive we do not observe a change in density during melting but the fluctuations in particle distances increases with decreasing $\Gamma$. This leads to a decrease of the maximum in $P(\Delta l)$ at $\Delta l=0$ and an increase of the maximal deviations of $\Delta l_{\max } \approx 20 \%$ at $\Gamma=132.9$ to $\Delta l_{\max } \approx 50 \%$ at $\Gamma=5.9$.

3. Similarly, the maximum of $P(|\Delta \Theta|)$ (at $|\Delta \Theta|=0^{\circ}$ ) decreases with increasing system temperature and the width of the distribution increases. In the crystalline phase at $\Gamma=132.9$, the maximal deviation (in sixfold space) is about $30^{\circ}$ (corresponding to $30^{\circ}$ in real space) whereas in the fluid phase the complete range of possible values $0^{\circ} \leq|\Delta \Theta| \leq 180^{\circ}$ is found.

The crossover in $P\left(m_{6}\right)$ for different $\Gamma$ is near $m_{6}=0.6$. This motivates to choose the threshold value to

$$
m_{T}=0.6 \text {. }
$$

In $P(\Delta l)$ the crossover is near $\Delta l \approx 10 \%$. This value is the full width at half maximum of curves close to melting $\left(\Gamma_{m}=60\right)$ and furthermore coincides with the the classical Lindemann criterion which states that a crystal melts, if the particle fluctuations exceeds $10 \%$ of the lattice distance. Therefore we choose $l_{T}$ to be

$$
\frac{l_{k l}-l_{a}}{l_{a}} \leq l_{T}=0.1
$$

The threshold for the bond orientation difference of nearby particles was defined as follows: the first two criteria were applied to configurational data of the crystal in equilibrium close to melting and $\Delta \Theta$ was increased from zero until the whole monolayer (except virtual dislocation pairs) were identified as monocrystal.

$$
\Theta_{T}=14^{\circ}
$$

This $14^{\circ}$ degree in sixfold space corresponds to $2.3^{\circ}$ in real space. Particles sitting in hexagons being less tilted with respect to each other are defined as crystalline cluster.

Figure 9 shows the number of particles in equilibrated systems which are identified to be crystalline. For comparison, the Larsen-Grier criterion $m+n>1$ and the number of particles with coordination number $C N=5,6,7$ is plotted, too. The Larsen-Grier criterion is slightly less tight but shows in principle the same result. Having a look at polycrystalline samples, grain boundaries seem to be made of two rows of particles 

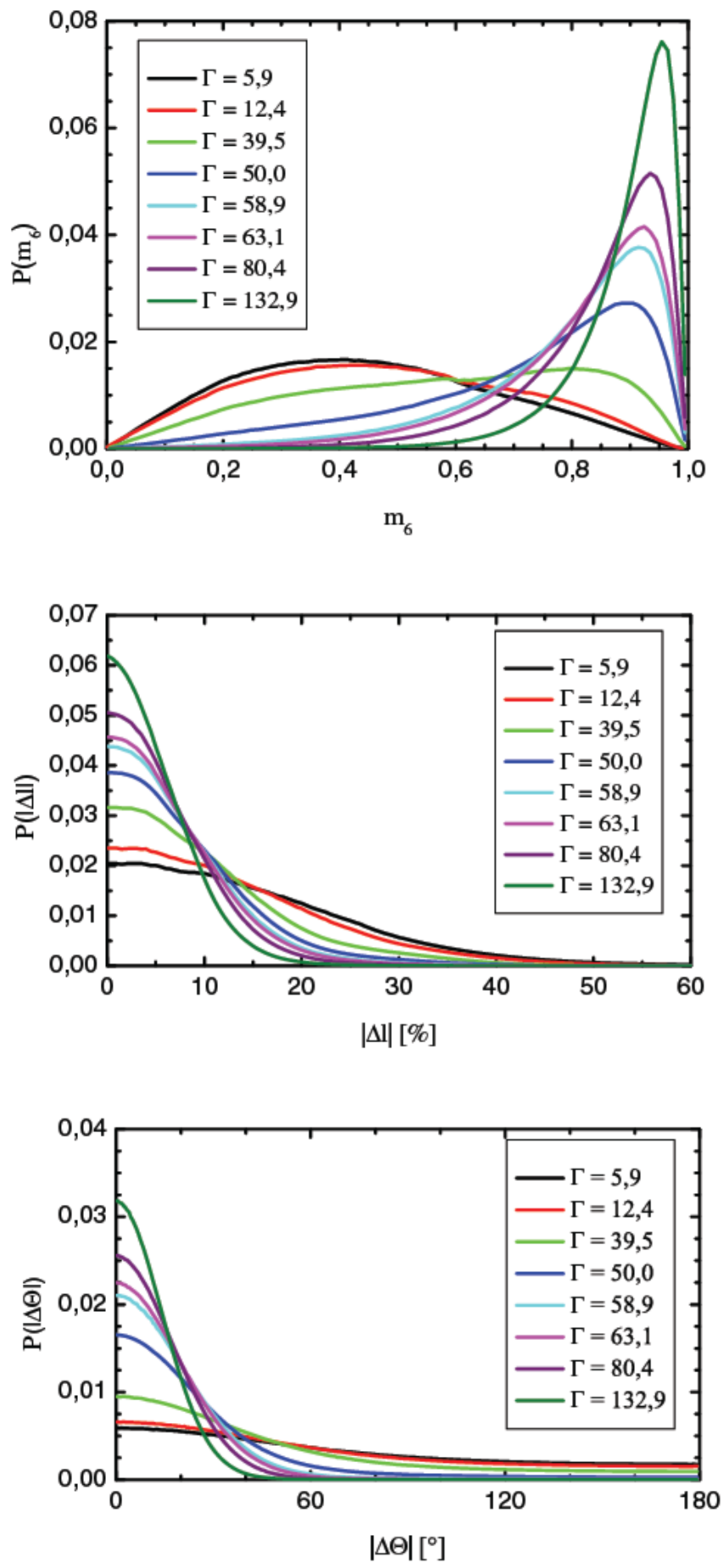

Fig. 8. Probability distribution as histogram for $P\left(m_{6}\right)$ to measure the hexagonal order (upper graph), distribution of the bond length deviation $P(\Delta l)$ (middle graph), and distribution of the difference of the bond orientation of neighboring particles $P(\Delta \Theta)$ for different interaction strengths of systems in equilibrium. 


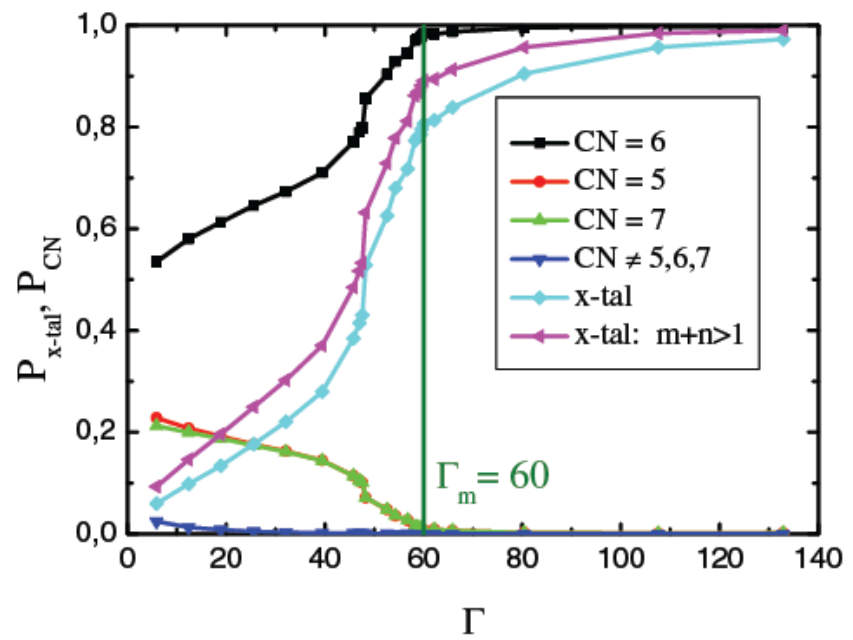

Fig. 9. Fraction of crystal like particles according to the three criteria including bond length variation and bond orientation difference compared with those determined using the magnitude of the bond order field projected to the second shell. Additionally, the fraction of 5,6 and 7 coordinated particles are shown as function of interaction strength.

with coordination number $C N \neq 6$ instead of one since the Larsen-Grier takes the second shell into account.

Close to melting, $80 \%$ of the particles are identified to be crystal-like, even if only $\approx 2 \%$ of the particle close to melting belong directly to virtual dislocation pairs, $20 \%$ are not crystal-like. Those are a) directly neighbours of dislocation pairs and b) looking at time series, they are precursors to dislocation pairs which appear several seconds later or c) residuals of virtual dislocation pairs, which has annihilated several seconds before [69]. Softening the criteria on the other hand would increase the number of crystal-like particles in the fluid phase. Even deep in the fluid phase still $10 \%$ of particles appear to be crystal-like. The lifetime of such 'precritical nuclei' is of the order of a few seconds and the average size is 2.7 particles. This large fraction of 'precritical nuclei' is attributed to the fact that the local close packed order in 2D is always sixfold: in the crystal as well as in the fluid. This additionally hints to the point that fluctuations in 2D, well known in equilibrium systems, play a major role in nonequilibrium transitions, too. [70].

\section{Cluster analysis}

After having identified crystal-like particles, those particles have to be merged into individual crystalline grains or cluster. This is done by giving a label to each crystallike particles which is identically within a cluster. Therefore we identify neighboring crystal-like particles $k$ and $l$ via Voronoi tessellation and check wether their bond order difference $\left|\Delta \Theta_{k l}\right|$ is again less than $\Theta_{T}=14^{\circ}$. The latter is necessary since crystal-like particles which belong to neighboring grains with different orientation should not be misinterpreted as belonging to the same grain. This way the grains (for a given time step) are scanned particle by particle and the overall bending of the lattice axis within a grain from one side to the other may exceed $\Theta_{T}=14^{\circ}$. Note, if this orientational criterion would be applied not relatively to the neighbors but absolutely in space, large and defect free monocrystals of equilibrated systems 
would not appear as such, since the bending of crystals in 2D due to Mermin-Wagner fluctuations is large.

If the crystalline cluster are identified for the whole duration of the experiment one can label the cluster of consecutive time steps to follow their temporal behaviour. Cluster of time $t_{i}$ and $t_{i+1}$ are compared by investigating the overlap in area of cluster $A_{j}$ at $t_{i}$ with $B_{k}$ at time $t_{i+1}$. The index $j$ ranges from 1 to $N_{t_{i}}$ and $k$ ranges from 1 to $N_{t_{i+1}}$ where $N_{t_{i}}$ and $N_{t_{i+1}}$ are the numbers of cluster in the time steps $t_{i}$ and $t_{i+1}$. The classification and correlation of the cluster is done as follows:

- No passing of Cluster-label $A_{j}$ to $B_{k}$ if no overlap in areas exists in the following time step. Cluster $A_{j}$ has disappeared.

- If cluster $A_{j}$ has only overlap with one cluster $B_{k}$, this cluster will become the label of cluster $A_{j}$ in the previous time step.

- If more than one cluster $B_{k}$ has overlap with $A_{j}$, the cluster with the largest overlap in area will become the label of $A_{j}$.

- If there are cluster $B_{k}$ which did not get a label after all cluster $A_{j}$ of time step $t_{i}$ has run through, they will get a new label.

The cluster analysis allows to follow the clusters in time and offers the possibility to investigate the area, circumference, shape and derivatives of such quantities in time. The only restriction is that clusters which are not completely in the field of view are neglected since their shape and size can not be determined correctly. This becomes relevant only at large rates of crystallinity and for late times where some clusters exceeds the field of view.

Figure 10 shows the crystalline clusters (here, cluster which touch the border are included) $10 \mathrm{~min}$ after a quench to $\Gamma_{E}=141$ (top) and $\Gamma_{E}=68$ (bottom) identified with the cluster analysis. For the deep quench almost the whole area is crystalline and only grain boundaries with a thickness of one to two particles and a view point defects in large grains are not crystalline. Note, that frequently one finds grains not separated by grain boundaries due to the strong bending of the lattice. The temporal development of patterns like in Fig. 10 (upper graph), which are almost completely crystalline, is very slow and can be described by Ostwald ripening [69]. For the lower quenches (e.g. lower plot), this is beyond our experimental time window but we were interested in the early times after a quench since new physics is expected in this range.

The large number of precritical nuclei in the fluid phase and the absence of any lack time after the quench indicates that the formation of a cluster is not a rare event. In this case, no nucleation barrier which defines a critical nucleus size has to be overcome by a fluctuation (in $2 \mathrm{D}$ a cost of energy in line tension is compensated by an energy gain in area). Hence one would expect all cluster which are present at the time of the quench to grow. But this is not what we observed: the probability of shrinking of individual clusters is always larger then the probability of growing, independent of the time after the quench, the size of the cluster and the depth of the quench [70].

\section{Fractal dimension}

The shape of the cluster and the ratio of circumference versus area provides information of the line tension of the clusters. In Fig. 11, we analyze for small grains with a size of up to ten particles the probability distribution $P\left(N_{V K}\right)$ of the circumference in units of Voronoi edges $N_{V K}$. A single sixfold particle has six Voronoi edges $N_{V K}^{1}=6$, two neighboring sixfold particles have $N_{V K}^{2}=2 *(6-1)=10$ since two edges of the two hexagons are connected/merged and do not count to the circumference. For three hexagons, two possibilities exist to arrange: as line then 

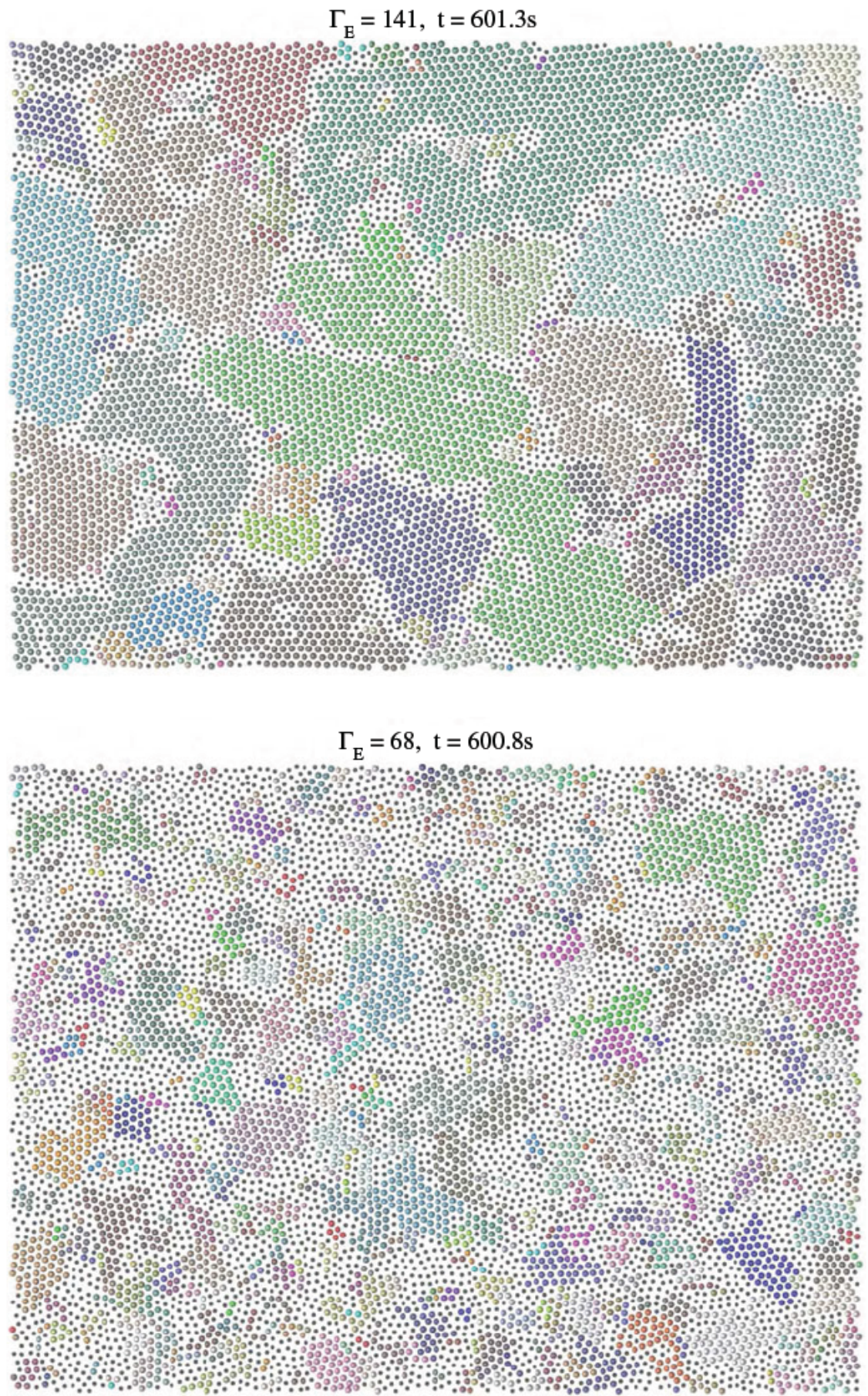

Fig. 10. Polycrystal $10 \mathrm{~min}$ after a quench to $\Gamma_{E}=141$ (top) and $\Gamma_{E}=68$ (bottom) identified with the cluster analysis. Same configurational data as in Fig. 3. 


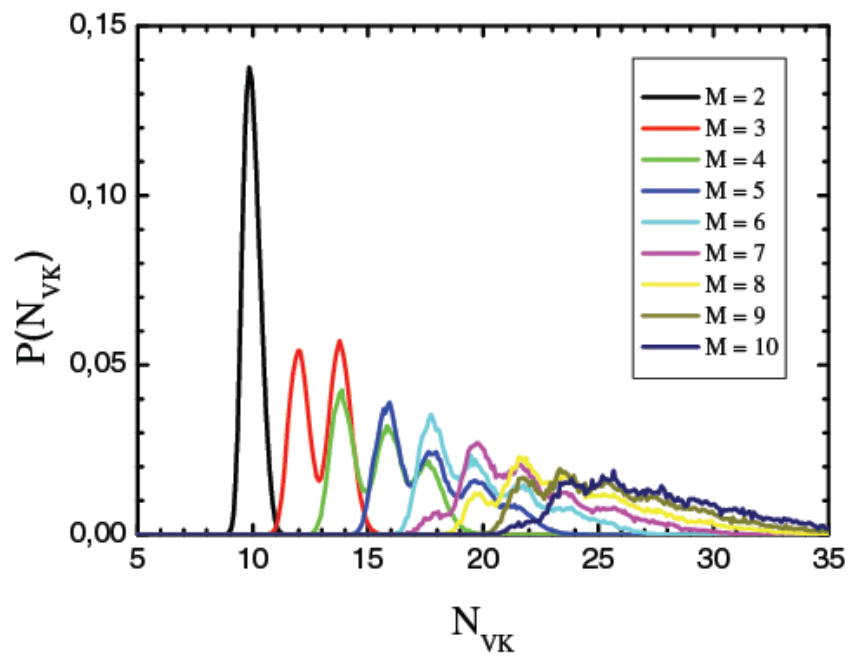

Fig. 11. Probability distribution of the circumference of the cluster $30 \mathrm{sec}$. after quench to $\Gamma=150$. The clusters do not necessarily minimize their circumference. Fluctuations lead to a broad distribution, indication a very small or even vanishing line tension.

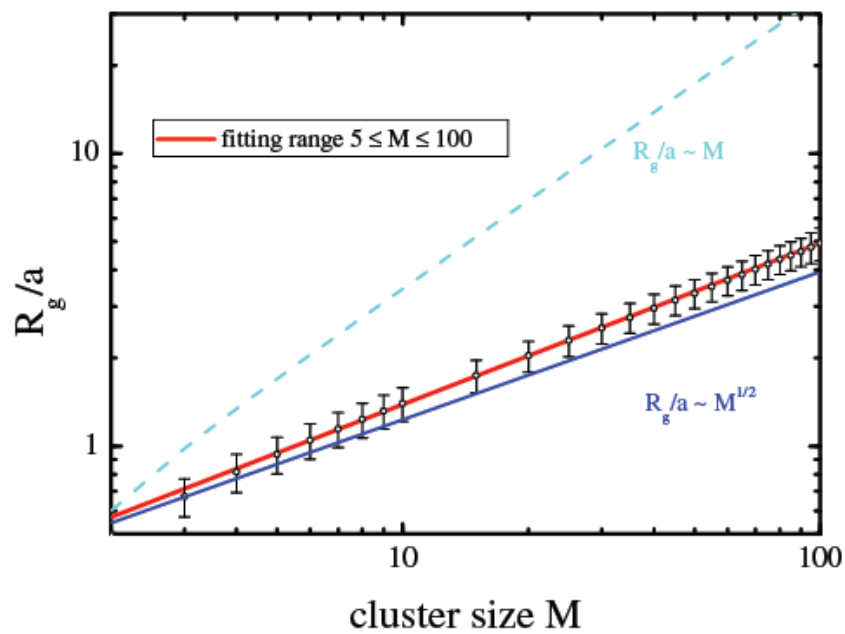

Fig. 12. Radius of gyration in a log-log plot as function of cluster size $10 \mathrm{~min}$ after a quench to $\Gamma_{E}=66$. The fractal dimensions is given by the slope of the fitting curve and is determined to be $d_{f}=1.8$.

$N_{V K}^{3}=3 * 6-2 * 2=14$ or as triangle, then $N_{V K}^{3}=3 *(6-2)=12$. For four hexagons, one finds three possible configurations like lines, L-shaped, and rhombic configurations. The analysis is performed $30 \mathrm{sec}$. after quench to $\Gamma=150$. One can see, that the configuration with the smallest circumference is not necessarily the most probable, even for the "magic-numbers" of seven particles, where the first shell may be closed.

To measure how spherical the clusters are, one might investigate the ratio of linear size (e.g. radius) to area. Following the ideas of fractal patterns, where fractal dimensions $d_{f}$ of scale invariant objects are (unlike topological dimensions $d_{T} \in N$ ) $d_{f} \in R$ and the fractal dimension can be used as measure of its roughness [72-75]. If $M$ is the number of particles (proportional to the area of the system) and $R_{g}$ is the 
radius of gyration one finds a power law

$$
M=k_{f}\left(\frac{R_{G}}{a}\right)^{d_{f}}
$$

where $k_{f}$ is a prefactor and $a$ is the lattice spacing [76]. The radius of gyration of cluster $j$ is given by

$$
R_{g}(j)=\sqrt{\frac{1}{m} \sum_{i=1}^{M} r_{i}^{2}}
$$

where $r_{i}$ is the distance of particle $i$ from the center of mass of cluster $j . R_{g}(M)$ is the mean of the radius of gyration of all clusters with particle number $M$. Figure 12 shows the radius of gyration in a log-log plot as function of cluster size $10 \mathrm{~min}$ after a quench to $\Gamma_{E}=66$. The red line is a fit to the data for $M \geq 5$ with a slope of $d_{f}=1.8 \pm 10^{-5}$. Dotted lines for one dimensional systems and purely spherical systems in $2 \mathrm{~d}$ are plotted for comparison, too. The fractal dimension is almost independent of the quench depth provided the fact that the system is not yet fully crystallized, e.g. $150 \mathrm{sec}$ after a quench to $\Gamma=63.3$ it is $d_{f}=1.8 \pm 10^{-5}$ and $30 / 150 \mathrm{sec}$ after a quench to $\Gamma=141$ it is $d_{f}=1.84 / 1.86 \pm 10^{-4}$, significantly below $d_{T}=2$.

\section{Conclusion}

Using a monolayer of super-paramagnetic particles we can perform ultra fast temperature quenches on time scales much faster compared to atomic systems. The crystallization is a kind of nucleation process without any indication of a hexatic phase known from equilibrated systems. KTHNY scenario, known from equilibrium systems, is not observed if the system is quenched rapidly below the melting temperature. The final structure is a polycrystal and even for the deepest quenches no amorphous phase is found. This might be explained by the fact that, unlike in $3 \mathrm{~d}$, the locally favored structures in the fluid are compatible with periodic order. The local order in $2 \mathrm{~d}$ is sixfold, in the fluid as well as in the crystal. This implies that the local bond order parameter $m_{6}$ is not sufficient to identify crystalline regions. We use additionally the bond length variation $\Delta l$ which has to be smaller than $10 \%$ and the criterion that neighboring hexagons are tilted less than $2.3^{\circ}$. Labeling cluster with maximal overlap of area in time, the time dependent behaviour of number, size, and shape of the cluster can be investigated. We observed the fractal dimension of the cluster to be $d_{f}=1.8$. As pointed out in [70] we do not find a critical nucleation barrier. In this case one expects that grains which are present at the quench should grow continuously - which is surprisingly not the case. Following individual grains in time the shrinking probability is always larger than the growing probability. This observation, together with the large number of 'precritical nuclei' in the fluid and the absence of a lack time indicates that classical nucleation theory which is compatible with a first order scenario does not describe the observed phenomena. 2D systems behave differently compared to $3 \mathrm{D}$ ones for both equilibrium and out of equilibrium situations.

This work was supported by the DFG within SFB TR6 (project C2).

\section{References}

1. R.E. Peierls, Ann. Inst. Henri Poincare 5, 177 (1935)

2. N.D. Mermin, Phys. Rev. 176, 250 (1968) 
3. N.D. Mermin, H. Wagner, Phys. Rev. Lett. 17, 1133 (1966)

4. V.M. Bedanov, G.V. Gadiyak, Y.E. Lozovik, Phys. Lett. A 109, 289 (1985)

5. X.H. Zheng, J.C. Earnshaw, Europhys. Lett. 41, 635 (1998)

6. S.T. Chui, Phys. Rev. Lett 48, 933 (1982)

7. S.T. Chui, Phys. Rev. B 28, 178 (1983)

8. H. Kleinert, Phys. Lett. A 95, 381 (1983)

9. M.A. Glaser, N.A. Clark, Adv. Chem. Phys. 83, 543 (1993)

10. Y. Lansac, M.A. Glaser, N.A. Clark, Phys. Rev. E 73, 041501 (2006)

11. J.M. Kosterlitz, D.J. Thouless, J. Phys. C 5, 124 (1972)

12. J.M. Kosterlitz, D.J. Thouless, J. Phys. C 6, 1181 (1973)

13. D.R. Nelson, J.M. Kosterlitz, Phys. Rev. Lett. 39, 2101 (1977)

14. D.J. Thouless, J. Phys. C 11, 189 (1978)

15. B.I. Halperin, D.R. Nelson, Phys. Rev. Lett. 41, 121 (1978)

16. D.R. Nelson, B.I. Halperin, Phys. Rev. B. 19, 2457 (1979)

17. A.P. Young, Phys. Rev. B 19, 1855 (1979)

18. B.J. Alder, T.E. Wainwright, J. Chem. Phys. 27, 1208 (1957)

19. K.J. Strandburg, Rev. Mod. Phys. 60, 161 (1988)

20. A. Jaster, Europhy. Lett. 42, 227 (1998)

21. J.J. Alonso, J.F. Fernandez, Phys. Rev. E 59, 2659 (1999)

22. S. Sengupta, P. Nielaba, K. Binder, Phys. Rev. E 61, 6294 (2000)

23. K. Binder, S. Sengupta, P. Nielaba, J. Phys. Cond. Mat. 14, 2323 (2002)

24. C.H. Mak, Phys. Rev. E 73, 065104 (2006)

25. S.Z. Lin, B. Zheng, S. Trimper, Phys. Rev. E 73, 066106 (2006)

26. E.P. Bernard, W. Krauth, Phys. Rev. Lett. 107, 155704 (2011)

27. C.A. Murray, D.H. Van Winkle, Phys. Rev. Lett. 58, 1200 (1987)

28. Y. Tang, A.J. Armstrong, R.C. Mockler, W.J. Osullivan, Phys. Rev. Lett. 62, 2401 (1989)

29. R.E. Kusner, J.A. Mann, J. Kerins, A.J. Dahm, Phys. Rev. Lett. 73, 3113 (1994)

30. A.H. Marcus, S.A. Rice, Phys. Rev. Lett. 77, 2577 (1996)

31. A.H. Marcus, S.A. Rice, Phys. Rev. E 55, 637 (1997)

32. K. Zahn, R. Lenke, G. Maret, Phys. Rev. Lett. 82, 2721 (1999)

33. K. Zahn, G. Maret, Phys. Rev. Lett. 85, 3656 (2000)

34. R.A. Segalman, A. Hexemer, R.C. Hayward, E.J. Kramer, Macromolecules 36, 3272 (2003)

35. D.E. Angelescu, C.K. Harrison, M.L. Trawick, R.A. Register, P.M. Chaikin, Phys. Rev. Lett. 95, $025702(2005)$

36. Y. Han, N.Y. Ha, A.M. Alsayed, A.G. Yodh, Phys. Rev. E 77, 041406 (2008)

37. Z.R. Wang, A.M. Alsayed, A.G. Yodh, Y.L. Han, J. Chem. Phys. 132, 154501 (2010)

38. H.H. von Grünberg, P. Keim, K. Zahn, G. Maret, Phys. Rev. Lett. 93, 255703 (2004)

39. P. Keim, G. Maret, H.H. von Grünberg, Phys. Rev. E 75, 031402 (2007)

40. S. Deutschländer, T. Horn, H. Löwen, G. Maret, P. Keim, Phys. Rev. Lett. 111, 098301 (2013)

41. P. Dillmann, G. Maret, P. Keim, J. Phys. Cond. Mat. 20, 404216 (2008)

42. R. Becker, W. Döring, Ann. Phys. 24, 719 (1935)

43. D. Turnbull, J.C. Fisher, J. Chem. Phys. 17, 71 (1949)

44. C.S. Kiang, D. Stauffer, G.H. Walker, O.P. Puri, J.D. Wise Jr., E.M. Patterson, J. Atmos. Sci. 28, 1222 (1971)

45. K. Binder, D. Stauffer, Adv. Phys. 25, 343 (1976)

46. D.W. Heerman, W. Klein, Phys. Rev. Lett. 50, 1062 (1983)

47. P.N. Pusey, W. van Megen, Nature 320, 340 (1986)

48. J. Zhu, M. Li, R. Rogers, W. Meyer, R.H. Ottewill, STS-73 Space Shuttle Crew, W.B. Russel, P.M. Chaikin, Nature 387, 883 (1997)

49. U. Gasser, E.R. Weeks, A. Schofield, P.N. Pusey, D.A. Weitz, Science 292, 258 (2001)

50. U. Gasser, A. Schofield, D.A. Weitz, J. Phys. Cond. Mat. 15, 375 (2003)

51. S. Auer, D. Frenkel, Nature 409, 1020 (2001)

52. V.J. Anderson, H.N. Lekkerkerker, Nature 416, 811 (2002) 
53. S. Auer, D. Frenkel, Adv. Polym. Sci. 173, 149 (2005)

54. D. Moroni, P.R. ten Wolde, P.G. Bolhuis, Phys. Rev. Lett. 94, 235703 (2005)

55. A. Cacciuto, S. Auer, D. Frenkel, Nature 428, 404 (2004)

56. L. Zheng, Q. An, Y. Xie, Z.H. Sun, S.N. Luo, J. Chem. Phys. 127, 164503 (2007)

57. V.I. Kalikmanov, J. Wölk, T. Kraska, J. Chem. Phys. 128, 124506 (2008)

58. T. Schilling, H. J. Schöpe, M. Oettel, G. Opletal, I. Snook, Phys. Rev. Lett. 105, 025701 (2010)

59. L. Granasy, G. Tegze, G.I. Toth, P. Tamas, Phil. Mag 91, 123 (2011)

60. T.H. Zhang, X.Y. Liu, J. Am. Chem. Soc. 129, 13520 (2007)

61. J.R. Savage, A.D. Dinsmore, Phys. Rev. Lett. 102, 198302 (2009)

62. P. Steinhardt, D.R. Nelson, M. Ronchetti, Phys. Rev. B 28, 784 (1983)

63. W. Lechner, C. Dellago, J. Chem Phys. 129, 114707 (2008)

64. F.C. Frank, Proc. R. Soc. Lond. A 215, 43 (1952)

65. C.P. Royall, S.R. Williams, T. Ohtsuka, H. Tanaka, Nature Mat. 7, 556 (2008)

66. F. Ebert, P. Dillmann, G. Maret, P. Keim, Rev. Sci. Inst. 80, 083902 (2009)

67. P. Dillmann, G. Maret, P. Keim, J. Phys. Cond. Mat. 24, 464118 (2012)

68. K.J. Strandburg, J.A. Zollweg, G.V. Chester, Pys. Rev. B 30, 2755 (1988)

69. P. Dillmann, Dissertation, http://kops.ub.uni-konstanz.de/handle/urn:nbn:de: bsz:352-196745

70. P. Dillmann, G. Maret, P. Keim, [arXiv:1303.6821] [cond-mat.soft]

71. A.E. Larsen, D.G. Grier, Phys. Rev. Lett. 76, 3862 (1996)

72. F. Hausdorf, Math. Ann. 79, 157 (1919)

73. A.S. Besicovitch, Math. Ann. 110, 321 (1935)

74. B.B. Mandelbrot, Science 156, 636 (1967)

75. L.F. Richardson, General Syst. Yearbook 6, 139 (1961)

76. C.M. Sorenson, J. Cai, N. Lu, Appl. Opt. 31, 6547 (1992) 